\title{
Application of Laser Line Scanners for Quality Control during Selective Laser Melting (SLM)
}

\author{
Kira Kristin Wehnert ${ }^{1}$, Sebastian Schäfer ${ }^{1}$, Jan Schmitt ${ }^{1}$, Andreas Schiffler ${ }^{1}$ \\ ${ }^{1}$ Institute Digital Engineering (IDEE), University of Applied Sciences Würzburg-Schweinfurt, \\ Ignaz-Schön-Str. 11, 97421 Schweinfurt, Germany \\ kirakristin.wehnert@fhws.de
}

\begin{abstract}
Summary
The use of laser line scanners for powder bed monitoring in 3D printing processes was investigated. It was possible to analyze dependencies in terms of exposure time due to the use of two different wavelengths of the laser line scanners. With a simplified setup - in accordance to a selective laser melting machine - the behavior of materials with nearly the same particle size as metal powder could be analyzed. In order to satisfy the conditions in the building chamber a construction platform was used. The behavior and the settings of the laser line scanner got evident, especially due to the strong reflections.
\end{abstract}

Keywords: metal printing, powder bed monitoring, quality control, laser line scanner

\section{Background, Motivation an Objective}

First time right is one major goal in powder based 3D metal printing. Reaching this goal is driven by reducing life cycle costs for quality measures, to minimize scrap rate and to increase productivity under optimal resource efficiency [1]. Therefore, monitoring the state of the powder bed for each printed layer is state of the art in selective laser melting. In the most modern approaches the quality monitoring is done by computer vision systems [2]. Nevertheless, the use of computer vision systems has drawbacks. Firstly, the sensor signals for the camera image do not provide depth information directly, which would correspond to a later height profile with height deviations. Secondly, the application of computer vision systems needs to be trained and labeled with reference images for several cases. This would require the images to be evaluated by human quality control or to be generated by image processing algorithms, which are highly sensitive to changing exposure situations. Therefore, it would be ideal to create a height profile per layer in order to be able to identify the severity of errors and, in the worst case, to stop the print job [1]. This not only saves material, time and costs, but also improves the process of the system.

\section{Description of the New Method or System}

The approach uses a laser line scanner to monitor the powder bed. With this new concept, several images during the print job of each layer can be taken and evaluated. The evaluation can be done online during the process and with feedback to the controller to monitor each indi- vidual layer. Hence, in case of deviations the location in the printing plane is an inherent measurement and will be used to decide which severity of error is reported [1]. With this approach, the sources of error for each layer can be analyzed with depth information to evaluate the cause of the error. Due to work safety during the development and the risks of the metal powder used, the tests with the laser line scanner were carried out on an external test setup. In principle, this is similar to the recoating process in the SLM (Selective Laser Melting) machine. Two different laser line scanners $\left(\lambda_{1 \text { (red }}\right.$ laser light) $=660 \mathrm{~nm} ; \lambda_{2 \text { (blue laser light) }}=405 \mathrm{~nm}$ ) are used for generating the profiles. In order to simulate metal powder without healthendangering risks, a similar powder with nearly the same particle size has to be utilized to exclude a risk regarding the health of the staff. Metal powder used for a SLM machine has a particle size between 10 and $45 \mu \mathrm{m}$ [2]. Flour and cocoa powder have proven to be the most suitable test material. The utilized flour has a particle size between 11-15 $\mu \mathrm{m}$ and the cocoa powder between 10-12 $\mu \mathrm{m}$. Both powders can be used for the experiments due to the similar particle sizes as replacement for metal powder.

\section{Results of the flour and the cocoa powder}

For the first measurements with the laser line scanners, the surfaces of the flour and the cocoa powder were smoothed. The results show that flour does not have a satisfactory surface layer, whereas the cocoa layer is satisfactory and can be used for further tests. The roughness of the cocoa layer was determined using 
white light interferometry. The arithmetic mean value $R_{a}$ is $25.8 \mu \mathrm{m}$. Based on this value a technical surface can be assumed where a speckle effect occurs. To compare the two sensors with each other, the process parameters are set and images of surface defects are generated. Since the exposure time and the surface condition of the measured surface have a significant impact on the sensor functionality, the experiments are carried out on cocoa powder layers and a reflective building platform with an already melted part. Another aspect why the mentioned platform is used is to get close to the environmental conditions in the building chamber. Because of the lighting in the chamber and trough reflective areas, additional external light can be caused.

\section{Results of the scanned cocoa powder}

In order to detect measurement artifacts caused by incorrectly set parameters, such as the exposure time, these are varied manually (Figure 1 , left). For example, the laser line scanner with red laser light produces underexposed areas at $400 \mu \mathrm{s}$ (Figure 1, middle) and overexposed areas at $2000 \mu \mathrm{s}$.
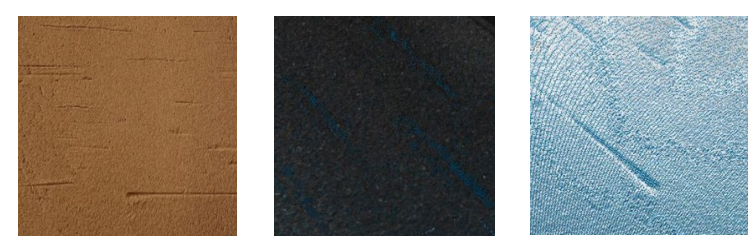

Figure 1 left: photo of the cocoa powder with caused creases; middle: red laser light underexposed areas at $400 \mu \mathrm{s}$; right: red laser light exposure at $1500 \mu \mathrm{s}$

The laser line scanner with the blue laser light has different error patterns. Defects due to underexposure appear after $240 \mu \mathrm{s}$ (Figure 2, left). By increasing the exposure time to $2600 \mu$ s (Figure 2, right), no defects caused by overexposure were found. By adjusting the exposure time exactly, it is possible to take images of powder layers with both sensors.
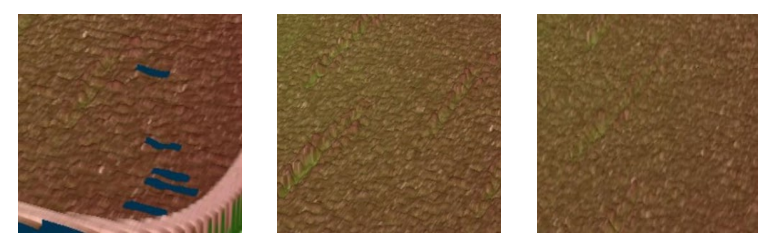

Figure 2 left: blue laser light underexposed areas at $240 \mu$ s; middle: blue laser light at $1700 \mu$ s; right: blue laser light at $2600 \mu \mathrm{s}$

\section{Results of the scanned building platform with red laser light}

The tests on the building platform are carried out with the same parameters that have already been used in the failure simulation. Significant defects occur at an exposure time of $1500 \mu \mathrm{s}$. Up to $4960 \mu \mathrm{s}$, defects increase due to overex- posure. Starting at an exposure time of $500 \mu \mathrm{s}$, defects continue to decline, but there are areas that are no longer sufficiently exposed. Defects disappear completely at $10 \mu$ s due to overexposure, but there are areas of the component that can no longer be detected. The results clearly show that reducing the exposure time reduces the amount of defects due to overexposure. It is possible to reduce the overexposure, but areas are still created that cannot be exposed sufficiently. It can be assumed that the sensor cannot provide meaningful measurement values for reflective areas that may occur in the production process.

\section{Results of the scanned building platform with blue laser light}

Since blue laser light can be used to acquire images from $960 \mu$ s onward, that do not show any errors due to overexposure or underexposure, only the smallest adjustable range of $15 \mu \mathrm{s}$ and the maximum adjustment range of $9600 \mu$ s were demonstrated. Errors caused by underexposure increase by reducing the exposure time. Increasing the exposure time eliminates the errors due to underexposure, but the errors caused by overexposure increase. The results clearly show that with the blue laser light at an optimal adjusted exposure time, images of reflecting surfaces can be captured without disturbing errors due to under- or overexposure.

\section{Final result and further outlook}

It was not possible to display reflective surface profiles using the red laser light, since errors occurred despite manual changes in exposure time. In contrast, an error-free display of the reflecting surface was achieved with the blue laser light. Therefore, it can be assumed that these are errors due to the speckle effect, which causes noise at the sensor receiver. Based on the investigations it seems that a lower wavelength of the blue laser light is more suitable for powder bed measurements. The results carried out for the approach to use laser line scanning for powder bed monitoring are promising. Additionally first feasibility tests within the real application confirm the results.

\section{References}

[1] Wehnert, K. K., Ochs, D., Schmitt, J., Hartmann, J., Schiffler, A., 2021. Reducing Lifecycle Costs due to Profile Scanning of the Powder Bed in Metal Printing. Procedia CIRP 98, 684-689. https://doi.org/10.1016/j.procir.2021.01.175.

[2] Grasso, M., Colosimo, B. M., 2017. Process defects and in situ monitoring methods in metal powder bed fusion: a review. Measurement Science and Technology 28, 044005. doi:10.1088/1361-6501/aa5c4f. 